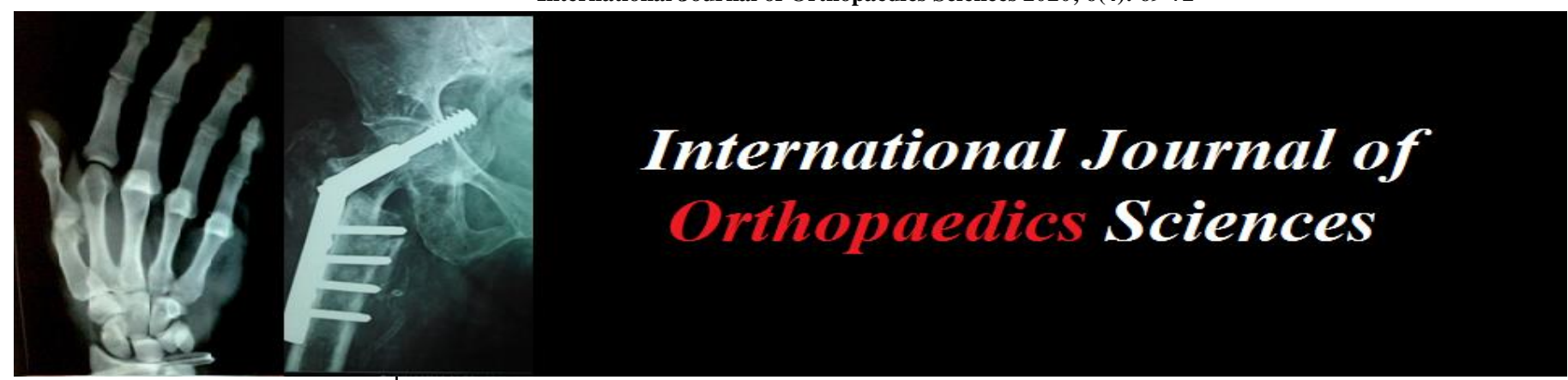

E-ISSN: 2395-1958

P-ISSN: 2706-6630

IJOS 2020; 6(4): 69-72

(C) $2020 \mathrm{IJOS}$

www.orthopaper.com

Received: 26-07-2020

Accepted: 03-09-2020

Dr. Kanakachalapathi Assistant Professor, Department of Orthopedics, Raichur Institute of Medical Sciences,

Raichur, Karnataka, India

Dr. Subhash Patil

Associate Professor, Department of Orthopedics, Raichur

Institute of Medical Sciences,

Raichur, Karnataka, India

\section{Comparative study of dynamic hip screw versus proximal femoral nailing in the management of intertrochanteric fracture femur}

\author{
Dr. Kanakachalapathi and Dr. Subhash Patil
}

DOI: https://doi.org/10.22271/ortho.2020.v6.i4b.2319

\section{Abstract}

DHS with side plate assembly is most commonly used device for fixation of intertrochanteric fractures. It is a non collapsible fixation device, which permits the proximal fragment to collapse or settle on the fixation device seeking its own position of stability. After the patient with intertrochanteric fracture was admitted to hospital all the necessary clinical details were recorded in proforma prepared for this study. After the completion of the hospital treatment patients were discharged and called for follow up at out patient level, at regular intervals for serial clinical and radiological evaluation. All patients were followed up at 2 weeks interval till fracture union, at 12 weeks and at 6 months post operatively. 5 patients failed to attend the first follow up and were lost for further follow up ( 3 cases of DHS and 2 PFN). At each follow up radiographs of upper femur and hip were taken to assess the fracture union, implant failure and screw cut out. In our series of 30 patients 5 cases were lost for follow up and 2 cases expired due to associated medical problems.

Keywords: Intertrochanteric fractures, DHS and PFN

\section{Introduction}

Intertrochanteric fractures are commonly seen in patients over 70 yrs of age. Incidence of these fractures has increased primarily due to increasing life span and more sedentary life style brought by urbanization. In younger population, IT fracture occurs due to high velocity trauma, where as in elderly population, it is most often due to trivial trauma.

Incidence of intertrochanteric fractures is more in females compared to males due to osteoporosis. Mortality ranges between $15 \%-20 \%{ }^{[1]}$.

IT fractures can be managed by conservative or operative methods. Conservative methods were the treatment of choice until 1960 before the introduction of new fixation devices. As conservative methods resulted in higher mortality rates and complications like decubitus ulcer, urinary tract infections, pneumonia, thromboembolic complications. These methods have been abandoned. Conservative methods are now indicated under 2 conditions,

i) Elderly person with high medical risk for anesthesia and surgery.

ii) Non ambulatory patient with minimal discomfort following injury ${ }^{[2,3]}$.

Rigid Internal fixation and early mobilization has been the standard method of treatment. Strength of fracture implant assembly depends on the following variables.

- Bone quality

- Fragment geometry

- Reduction

- Implant type

- Implant placement.

Surgeon can control only the quality of reduction, choice of implant and its placement ${ }^{[4]}$. Implants for the fixation of intertrochanteric fractures can broadly be divided into

- Extramedullary devices, ex:-.DHS.

- Intramedullary devices ex:-PFN,

\section{Dr. Subhash Patil}

Associate Professor, Department

of Orthopedics, Raichur

Institute of Medical Sciences,

Raichur, Karnataka, India 
DHS with side plate assembly is most commonly used device for fixation of intertrochanteric fractures. It is a non collapsible fixation device, which permits the proximal fragment to collapse or settle on the fixation device seeking its own position of stability ${ }^{[5]}$.

The latest implant for management of intertrochanteric fracture is PFN. This implant is a cephalomedullary device and has many potential advantages like ${ }^{[6]}$.

- Being intramedullary, load transfer is more efficient.

- Shorter lever arm results in less transfer of the stress and hence less chance of implant failures.

- Advantage of controlled impaction is maintained.

- Amount of sliding is limited by intramedullary location, therefore less chance of shortening and deformity.

- Shorter operative time, less soft tissue dissection and less blood loss.

- In view of these conditions, this study is taken up to compare the results of DHS and PFN in the treatment of intertrochanteric fractures.

\section{Methodology}

The present study consists of 30 adult patients with intertrochanteric fractures of femur who were treated with DHS and PFN admitted to department of orthopaedics, Raichur Institute of Medical Sciences, Raichur, from dec 2017 to dec 2018. Patients with more than 20yrs, stable and unstable intertrochanteric fractures are included in this study.

This study was carried out to study the results of intertrochanteric fractures treated with DHS and PFN.

\section{Data collection}

After the patient with intertrochanteric fracture was admitted to hospital all the necessary clinical details were recorded in proforma prepared for this study. After the completion of the hospital treatment patients were discharged and called for follow up at out patient level, at regular intervals for serial clinical and radiological evaluation.

\section{Management of patient}

As soon as the patient with suspected intertrochanteric fracture was seen, necessary clinical and radiological evaluation was done and admitted to ward after necessary resuscitation and splintage with skeletal traction.

The following investigations were done routinely on all these patients preoperatively.

Blood; $\mathrm{Hb} \%$
Bleeding time
Clotting time
Blood grouping and Cross matching
Fasting and Post prandial blood sugar
Blood urea and Serum Creatinine

Urine: Albumin

Sugar

Microscopic examination

X-ray;

Pelvis with both hips AP view

Chest $\mathrm{X}$ ray $\mathrm{PA}$ view in necessary patients

All the patients were evaluated for associated medical problems and were referred to respective department and treated accordingly.

Associated injuries were evaluated and treated simultaneously. The patients were operated on selective basis after overcoming the avoidable anaesthetic risks.

\section{Results}

In our study maximum age was 85 years and minimum age was 20 years. Most of the patients were between 25-60 years. Mean age was 48.6 years.

In our study the average duration of hospital stay was 26.13 days for DHS patients and 26.8 days for PFN patients.

The mean time of full weight bearing was 10.6 weeks for PFN and 14.8 weeks for DHS.

All patients enjoyed good, hip and knee range of motion except for 1 patient of PFN who had extensive lateral cortex communition during surgery and had to be immobilized for prolonged period.

All the patients except 1 case of PFN was ambulatory with or without walking aid at 6 weeks follow up.

All patients were followed up at 2 weeks interval till fracture union, at 12 weeks and at 6 months post operatively.

5 patients failed to attend the first follow up and were lost for further follow up ( 3 cases of DHS and 2 PFN). At each follow up radiographs of upper femur and hip were taken to assess the fracture union, implant failure and screw cut out.

In our series of 30 patients 5 cases were lost for follow up and 2 cases expired due to associated medical problems.

Functional and Anatomical results were assessed by taking the remaining 23 cases into consideration.

Table 1: Age distribution

\begin{tabular}{|c|c|c|c|c|}
\hline Age group & \multicolumn{2}{|c|}{ Number of cases } & \multicolumn{2}{c|}{ Percentage } \\
\hline & PFN & DHS & PFN & DHS \\
\hline $0-20$ & 1 & 1 & $3.33 \%$ & $3.33 \%$ \\
\hline $21-40$ & 6 & 3 & $19.99 \%$ & $9.99 \%$ \\
\hline $41-60$ & 4 & 5 & $13.33 \%$ & $16.65 \%$ \\
\hline $61-80$ & 2 & 6 & $16.67 \%$ & $19.99 \%$ \\
\hline $81-100$ & 2 & 0 & $16.67 \%$ & 0 \\
\hline Total & 15 & 15 & $50 \%$ & $50 \%$ \\
\hline
\end{tabular}

Table 2: Assesment of results

\begin{tabular}{|c|c|c|}
\hline & PFN & DHS \\
\hline Mean duration of Hospital stay (in days) & 26.8 & 26.13 \\
\hline Mean time for full weight bearing (in weeks) & 10.6 & 14.8 \\
\hline Mobility after surgery(6 weeks post operatively) & & \\
\hline Independent & 9 & 7 \\
\hline Aided & 3 & 5 \\
\hline Non-ambulatory & 1 & 0 \\
\hline Mean range of movements (6weeks post operatively) & & \\
\hline Hip-joint(0-110 degrees) & $12 / 13$ & $12 / 12$ \\
\hline Knee joint(0-120 degrees) & $12 / 13$ & $12 / 12$ \\
\hline
\end{tabular}

Table 3: Anatomical Result

\begin{tabular}{|c|c|c|}
\hline Anatomical Result & \multicolumn{2}{|c|}{ Number of cases } \\
\hline & PFN & DHS \\
\hline Shortening more than 1cm & 2 & 1 \\
\hline Varus deformity & 2 & 1 \\
\hline Restriction of Hip movement & 2 & 4 \\
\hline Restriction of Knee movement & 1 & 0 \\
\hline
\end{tabular}




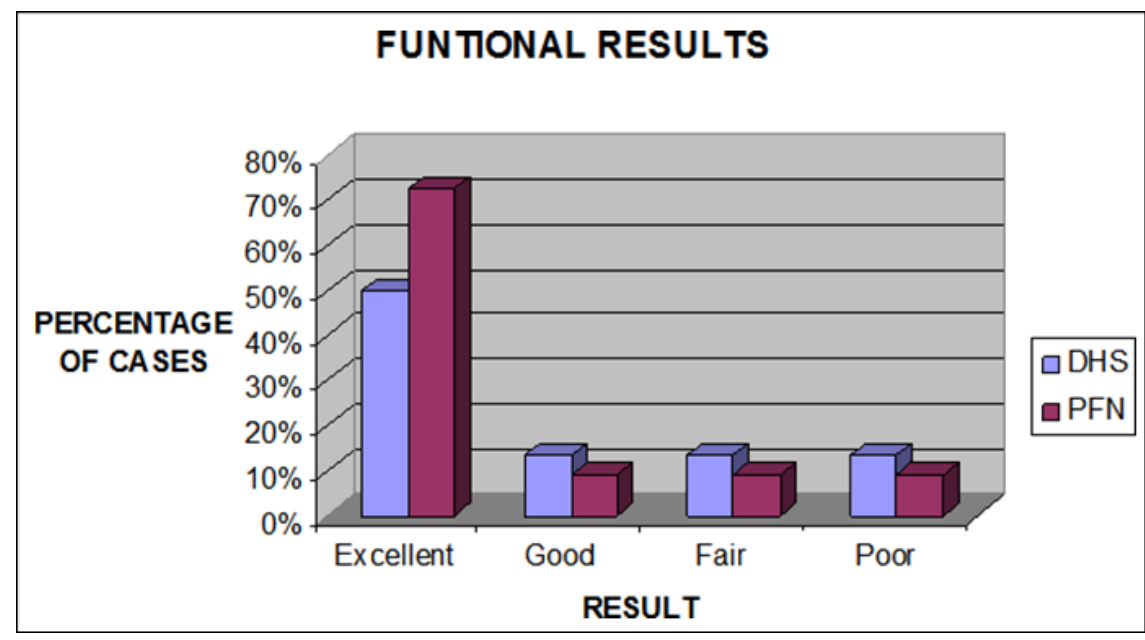

Graph 1: Functional results of PFN and DHS

\section{Discussion}

The most commonly used method of fixation is sliding screw system. From mechanical point of view an intramedullary device inserted by means of minimally invasive procedure seems to be better in elderly patients. Closed reduction preserves the fracture heamatoma, an essential element in consolidation process. Intramedullary fixation allows the surgeon to minimize soft tissue dissection, there by reducing surgical trauma, blood loss, infection and wound complications [7].

PFN is a novel, modern, intramedullary implant based on experience with Gamma nail. The currently used Gamma nail as an intramedullary device has high learning curve with technical and mechanical failure rates of about $10 \%$. The Gamma nail is susceptible to fail at its weakest point, the lag screw -implant interface ${ }^{[8,9]}$.

The AO ASIF in 1996, therefore developed the Proximal Femoral Nail with an antirotation hip pin together with a smaller distal shaft diameter which reduces stress concentration to avoid these failures. PFN has all the advantages of an intramedullary device such as decreasing the moment arm, can be inserted by closed technique which retains the fracture heamatoma, decreases blood loss, minimizes soft tissue dissection and wound infections.

Menez and Daneil conducted a study on 155 cases of intertrochanteric fractures treated with $\mathrm{PFN}$, and found $2 \%$ failure of fixation and $0.7 \%$ of femur shaft fractures. However no such complications was reported in our study ${ }^{[10]}$.

In a study, by Pajarein and Lindal, of 108 patients of pertrochanteric fractures treated with DHS and PFN, found PFN allowed faster restoration of post-operative walking ability when compared with DHS ${ }^{[11]}$. In our study mean time for full weight bearing for DHS was 14.8 weeks while that for PFN was 10.6 weeks. 9 of 13 patients treated with PFN had indepent mobility while 7 of 12 patients of DHS had independent mobility.

A study of 20 patients of unstable intertrochanteric fractures treated with PFN and DHS by Barathi and Arshad, was conducted in $2004^{[12]}$. They found duration of stay for PFN and DHS were 14 and 22 days, blood loss was 275 and $475 \mathrm{ml}$, persistent hip pain was seen in $3 \%$ and $9 \%$ respectively. This correlated with our study where the duration of hospital stay was 26 days in both cases, average blood loss was 240 and 320 $\mathrm{ml}$, persistent hip pain was seen in $1 / 11$ and 2/12 cases of PFN and DHS respectively.

In our study, intertrochanteric fracture was common due to fall from height, age ranged between 16-85 years, mean age of 48.6 years. Females were common contributing to $64 \%$. Right sided fractures were common accounting for $70 \%$.

In our study, Type II Boyd and Griffin fractures were common, consisted of $53.3 \%$. Type I and Type III were $30 \%$ and $16.6 \%$ respectively.

Mean frequency of radiaton exposure were 70 and 40 times mean duration of duration of operation 100 and 80 minutes, mean blood loss was $240 \mathrm{ml}$ and $320 \mathrm{ml}$ for PFN and DHS respectively.

Intraoperatively 7 cases had to undergo open reduction, iatrogenic fracture of lateral cortex was seen in 3 cases, 3 cases fracture got displaced by nail insertion, 4 cases antirotaton screw could not be put in PFN group.

Among DHS, intraopertively had fewer complications which included improper placement of the screw in 5 cases, varus angulation in 3 cases, drill bit breakage in 1 case.

Post operatively 1 case of PFN had wound infection and none of the DHS cases got infected.

Mean duration of hospital stay was 26 days in both cases, mean time of full weight were 10.6 and 14.8 in PFN and DHS. All patients were mobile at the end of 6 weeks with or without walking aid except for one case of PFN. 9/13 and 7/12 cases of PFN and DHS had independent mobility

In our 30 cases, 5 patients were lost for follow up and 2 cases died due to associated medical problems.

Excellent results were seen in $50 \%$ and $72.73 \%$, good in 13.33 and $9.1 \%$ cases of DHS and PFN respectively.

\section{Conclusion}

From the study, we consider PFN as better alternative to DHS in the treatment of intertrochanteric fractures but is technically difficult procedure and requires more expertise compared to DHS. With experience gained from each case operative time, radiation exposure, blood loss and intraoperative complications can be reduced in case of PFN

\section{References}

1. Terry Canale's S. Campbell's Operative Orthopedics, Tenth Edition, 3, 2874-2897.

2. Micheal W. Chapman's Chapman's Orthopaedic Surgery, Third Edition, Lipincott Williams And Wilkins Company, 1, 634-669.

3. Habernek H, Wallnce $\mathrm{T}$, Aschaner E, Schmid L. Comparision of Ender's Nails, Dynamic Hip Screw and Gamma Nails in Treatment of Peritronteric Femoral Fractures Orthopaedic. 2000; 23(2):127(7).

4. Leung KS et al. 186 Patients 136 Unstable Fractures A 
Randomized Prospective Study JBJS [B]. 1992;784B345-51.

5. Ruland WD. Pertrochanteric Femoral Fractures in Elderly Patients-Injury Pattern and Management with Gamma Nailing. Langenbecks-Arch-Chir-Suppl- Kongresshd. 1996; 113:996-8.

6. Saudan Marc, Lübbeke Anne, Sadowski Christophe, Riand Nicolas, Stern Richard, Hoffmeyer Pierre. Is Pertrochanteric Fractures: There An Advantage To An Intramedullary Nail?: A Randomized, Prospective Study Of 206 Patients Comparing The Dynamic Hip Screw And Proximal Femoral Nail. 2002; 16(6):386-393.

7. Michael Baumgaertner R, Thamos Higgins F. Chapter 38 In Rockwood And Green's Fractures in Adults. $5^{\text {th }}$ Edition. 2, 1579-94, 1665-1681.

8. Mcconnell. Alison Msc (Eng)*; Zdero, Rad Phd*; Syed, Khalid Md, The Biomechanics Of Ipsilateral Intertrochanteric And Femoral Shaft Fractures: A Comparison Of 5 Fracture Fixation Techniques. 2008;22(8):517-524.

9. Simmermacher RKJ, Bosch AM, Van Der Werken C. The Ao Asif-Proximal Femoral Nail (PFN): A New Device for the Treatment of Unstable Proximal Femoral Fractures Injury. 1999; 30:327-32.

10. Menes Daniel, Gamulin Noesberg. Is Proximal Femoral Nail A Suitable Implant for Treatment of All Trochanteric Fractures Lipincott, Williams and Wilkins Inc. 2005;439:221-227.

11. Pajarinen J, Lindahl J, Michelsson O, Savolainen V, Hirvensalo E. Pertrochanteric Femoral Fractures Treated With A Dynamic Hip Screw Or A Proximal Femoral Nail: A Randomised Study Comparing Post-Operative Rehabilitation. 2005;87-B(1):76-81.

12. Bhatti Arshad, Power Dominic, Qureshi Sohail, Khan Ishrat, Tan Simon. A Prospective Trial of Proximal Femoral Nail versus Dynamic Hip Screw for Unstable and Complex Intertrochanteric Fractures of the Femur. 2004; 86-B (Supplement III):377. 